\title{
EL FASTO IMPERIAL Y LOS MILIARIOS EN EL SIGLO III. LA PRESENCIA DE LAS AUGUSTAE
}

\author{
THE IMPERIAL GRANDNESS AND MILESTONES IN THE THIRD CENTURY A. D. \\ THE PRESENCE OF AUGUSTAE
}

\author{
RAFAEL GONZÁLEZ FERNÁNDEZ \\ Universidad de Murcia \\ rafaelg@um.es \\ http://orcid.org/0000-0002-7758-8565
}

Recepción: 30-10-2016

Aceptación: 01-02-2017

\section{Resumen}

Los miliarios realizaban en el mundo romano una doble función, por una parte, como indicadores de rutas al servicio del viajero y por otra como elementos de representación política. En época imperial suponían un claro homenaje al emperador y a su familia como representación del poder. A partir sobre todo de la dinastía de los Severos, se asiste a la aparición de titulaturas imperiales en cualquier clase de obra pública, incluidos los miliarios que se convierten de esta manera en un ejemplo claro de soporte de la proyección del poder político. En este caso analizamos aquéllos en los que como reflejo de este hecho aparecen mujeres de la familia imperial, que además son Augustas, junto a otros miembros de la domus imperial, durante el siglo III d.C., único momento en el que tal práctica se lleva a cabo.

Palabras clave. Emperatrices; representación política; familia imperial; Imperio Romano; dinastía de los Severos; titulatura imperial.

\begin{abstract}
The milestones in the Roman world performed a dual function, firstly as indicators of the routes at the traveler's service, and secondly as elements of political representation. During the Imperial age, they were a clear homage to the emperor and his family as a representation of power. Especially from the Severan dynasty onwards, the imperial titulature in any kind of public work appears, including milestones that thus become a clear example of support for political power propaganda. Here we analyze the cases in which, as a consequence of the mentioned fact, we see the presence of women of the imperial family, who are also Augustae, along with other members of the imperial household. The third century is the only period in which such practice is performed.
\end{abstract}

Key words. Empresses; political representation; imperial family; Roman Empire; Severan dynasty; imperial titulature. 


\section{INTRODUCCIÓN}

Los miliarios que fueron colocados a lo largo de las viae publicae son un elemento clave de la red de carreteras del Imperio Romano. Estos monumentos, que ahora suman entre siete y ocho mil (Kolb, 2015: $654)^{1}$, indicaban la distancia desde el caput viae, pero además sus textos no sólo proporcionan información relacionada con la administración y organización de la red de carreteras, sino que a su vez también representan importantes evidencias de la administración provincial, del poder del emperador y de la cultura romana (Kolb, 2011: 17; Kolb, 2015: 654). Son verdaderos documentos oficiales que, colocados a lo largo de las vías, constituyen una expresión de la política estatal, bien sea de los desplazamientos de los emperadores, bien se trate de programas económicos o militares (Chevallier, 1999: VII). Así, viajando a través de las calzadas romanas se podía encontrar el nombre del emperador en cada uno de estos hitos que, junto a edificios y estatuas, ayudaban a hacerlo omnipresente (Santosuosso, 2001: 111). Constituyen además una categoría específica de los llamados tituli operum locorumque publicorum (Beltrán Lloris, 2015: 94; Kolb, 2015: 656) y se incluyen en un volumen independiente del Corpus Inscriptionum Latinarum (CIL XVII), aún en curso de publicación. Aunque es relativamente habitual referirse en la historiografía arqueológica o en la propiamente histórica a la propanda imperial, sin embargo, el término y el concepto mismo de propaganda es impropio cuando se utiliza para la Antigüedad (Arce, 2007: 3). Concretamente, en un artículo en relación con las monedas de época imperial, B. Levick (1982) demostró que hablar de propaganda en época romana resulta anacrónico. De igual forma, P. Veyne sostiene que la palabra propaganda suena históricamente falsa, pues en lugar de comprender mejor los hechos antiguos acercándolos al presente, ignora la mentalidad de antaño, llegando este mismo autor a la conclusión de que es de aparato, de fasto monárquico de lo que hay que hablar (Veyne, 2009: 336).

Desde un punto de vista morfológico integran uno de los tipos de soporte epigráfico más estables en el mundo romano (Cagnat, 1914: 72-176; Calabi, 1968: 285; Moreno, 2004: 158-162; Andreu Pintado, 2009: 426). Para J. M. Lassère esto es así por su naturaleza 'propagandística' y su estrecha relación con la titulatura imperial que preside su texto (Lassère, 2005: 919). Asimismo, los considera la marca de la autoridad estatal en el paisaje, ya que son un importante testimonio en la vida del Estado y la manifestación evidente de la autoridad central en las provincias

1. Para otras cifras menores, en torno a cinco mil, $c f$. Schneider (1982: 103), Bender (1989: 119) y van Tilburg (2007: 20).
(Lassère, 2005: 917)². Además, constituyen lo que Giancarlo Susini ha llamado «pedagogía del poder»; según este autor en época tardoantigua se exponen titulaturas complejas que justificarían su conversión solamente con un fin, como exposición de una apología del emperador, esto es, como un auténtico manifiesto de consenso político (Susini, 1982: 120 y 125). Asímismo, presentan uno de los formularios más interesantes y variables de la epigrafía romana que, a pesar de su aparente sencillez, sin embargo, han permitido establecer diferentes tipologías (Cagnat, 1914: 172-176; Calabi, 1968: 285). Precisamente entre los textos de las obras públicas, el de los miliarios es quizás el que más cambios ha sufrido en su evolución a lo largo del tiempo, tanto en su estructura como en su léxico, con la finalidad principal y ya comentada, de servir como elemento de representación política (Donati, 2002: 33). En época republicana los primeros miliarios tenían un formulario muy simple: mencionaban el nombre del magistrado responsable, normalmente pretor o cónsul, de la construcción o la restauración de la vía (aunque en ocasiones no es fácil distinguir entre una y otra) y la indicación, con la distintiva abreviatura $m$ (illia) $p$ (asuum) aunque no siempre expresada, de la longitud del tramo entre dos ciudades, desde el punto en que era colocado, siendo esta última la que verdaderamente subrayaba la función de indicador viario del monumento. Con Augusto, después de un paréntesis, su uso fue reintroducido a partir del 20 a.C. en adelante y desarrollado fundamentalmente como un instrumento de representación reservado para el emperador (Alföldy, 1991: 299-302). De esta forma, hasta la mitad del siglo III d. C. continúan siendo registrados estos dos últimos elementos, con todas las variantes derivadas de la presencia de la rica titulatura imperial. En definitiva, en época imperial el formulario se enriquece. Presenta el nombre del emperador designado con una titulatura muy completa y sobre todo caracterizada por la tribunicia potestas que permite precisar su cronología. Normalmente el nombre del emperador figura en nominativo como cualquier otro elemento emanado de su autoridad (Cooley, 2012: 159-162). A partir de la dinastía de los Severos, y sobre todo en África, se hace mucho más hincapié en la función conmemorativa por el hecho de que ahora los nombres y títulos del emperador y miembros de la familia imperial, son expresados en dativo como cualquier dedicatoria honorífica, encargada por los responsables de la gestión y mantenimiento de las vías ${ }^{3}$ (Noreña, 2011: 220), si bien es verdad que al menos desde Adriano ya hay miliarios con su nombre y títulos en dativo. Se

2. También sobre el papel del Estado en los diferentes espacios provinciales $c f$. Nicolet (1988).

3. Sobre las variaciones en los casos gramaticales de las inscripciones, particularmente en el África Proconsular, $c f$. Salama (1987: 56-60 y 131-135). 
convierten así en elementos omnipresentes del fasto oficial $^{4}$ y la epigrafía miliaria viene a tomar, en cierto sentido, el relevo de la del foro ${ }^{5}$ (Lassère, 2005: 918). Precisamente se ha sugerido que en algunas zonas del Imperio (v.g. Baetica), en el siglo IV d. C. los caminos habrían reemplazado al foro como escenario principal de la conmemoración epigráfica de los emperadores (Sillières, 1986). En definitiva, junto con las propias carreteras los miliarios proyectaron una imagen del control de Roma sobre todo el territorio del Imperio al menos hasta 435 d.C. en Occidente y 521 d. C. en Oriente (Kolb, 2004: 142-144).

\section{LOS MILIARIOS COMO VEHÍCULO DE LA REPRESENTACIÓN IMPERIAL ${ }^{6}$}

Es a partir del último emperador Antonino cuando los miliarios empiezan a presentar lo que C. F. Noreña ha denominado terminología honorífica (Noreña, 2011: 220), normalmente epítetos superlativos dedicados al emperador en este tipo de inscripciones oficiales. El más antiguo conocido es de 186 d. C. y está dedicado, aún en nominativo, a Cómodo en Numidia $(C I L$ VIII, $10307=I L S$ 397). La anomalía radicaría en el uso de estos epítetos sobre monumentos con inscripciones para los cuales el estado normalmente asumía la responsabilidad. Terminología que no aparece en otros textos oficiales emanados de la propia cancillería imperial (v.g. diplomas militares) por lo que algunos investigadores los consideran títulos no oficiales (Frei-Stolba, 1969). Se podría pensar en iniciativas de los gobernadores o de las propias autoridades locales, que seguramente no desagradarían al emperador (Christol, 1999: 351-352). Otro argumento a favor de esta interpretación es precisamente el uso cada vez mayor del dativo en los nombres y títulos, lo que implicaría que estos monumentos oficiales podrían verse como un homenaje al monarca. En general podemos decir que estas obras públicas se construyen por voluntad y en nombre del emperador; su nombre y títulos lo muestran como responsable de la obra a lo largo de todos los caminos y vías; los miliarios repiten las alabanzas a intervalos fijos, con la doble función de informar al viajero sobre el camino que lleva recorrido o le queda, y de recordarle el nombre y las cualidades del emperador, por supuesto esto también tenía implicaciones militares, pues era

4. Sillières (1986) y Salama (1992) hablan del miliario como instrumento de propaganda. El segundo autor analiza una colección procedente de Numidia que datan de los años 319320. Comparados con las emisiones monetarias muestran cómo han sido utilizados como instrumentos de la política imperial; d'Encarnação (1995-1996).

5. Esta misma idea fue manifestada por Christol (1999: 352).

6. Pekary (1968: 16-22); König (1973); Kuhoff (1990); Kuhoff (1993a); Ando (2000: 322-323); Witschel (2002); Kolb (2004); Noreña (2011: 220-223). el ejército hasta ese momento quien supervisaba y llevaba a cabo la construcción de carreteras donde quiera que estuvieran (Levick, 2007: 68). Sin embargo, poco a poco, con el paso del tiempo, se va asistiendo a una gradual y progresiva desaparición de la indicación numérica; asimismo se va transformando la propia titulatura imperial que pierde la referencia a los cargos institucionales propios del cursus honorum, y se va enriqueciendo con elementos que alaban las virtudes y la capacidad del emperador. Expuestos en los márgenes de las calzadas romanas, destinados a la lectura de un público vasto y diversificado, y aunque formalmente siguen ejerciendo su función como textos informativos que marcan las distancias viarias, sin embargo, en ellos prevalece, sobre todo a partir de la dinastía severiana, el papel de instrumento de comunicación o también de acto de homenaje al emperador y a la familia imperial (Donati, 2002: 33-35; Alves Dias, 2015: 95). Incluso a partir del reinado de Constantino, de forma cada vez más frecuente se omiten las distancias y la información que se ofrece a los viajeros se centra en los formularios que reúnen los numerosos títulos de los emperadores, incluso los considerados usurpadores, que los utilizan como expresión de su poder.

\section{LOS MILIARIOS EN EL SIGLO III}

A partir de Septimio Severo se producen cambios significativos en la estructura de los formularios de los miliarios, iniciando una serie de novedades que serán continuadas en los siglos siguientes (Christol, 1999: 350-353). Por tanto, del siglo III d. C. en adelante hubo notables transformaciones. Pasaron de ser semejantes a las inscripciones monumentales a hacerse más afines a las dedicatorias o monumentos honoríficos. A partir de ahora incluso son emplazados por una ciudad y dedicados al emperador (Levick, 2000: 115). Aunque éste sigue siendo mencionado en nominativo, como la fuente última de la obra que promovió ésta, su nombre, como ya hemos mencionado más arriba, aparece cada vez con más frecuencia en dativo. Pero este cambio no ocurre simúltaneamente en todo el Imperio, sino que es introducido gradualmente a partir de este siglo, convirtiéndose en la norma durante el siglo IV d. C. (Sillières, 1986: 356-357; Witschel, 2002: 327, 332 y 335; Cooley, 2012: 163). Como resultado de ello, el texto toma la forma habitual de una dedicatoria. Por medio de este tipo de «monumentos-dedicatorias», que por definición se erigen en muchos lugares muy frecuentados, en las provincias, las comunidades de todo el Imperio aparecen, cada vez más, honrando al emperador y manifestando su lealtad a la dinastía reinante (Isaac, 1993: 304-309; Christol, 1999: 352). Independientemente de que se hubiera realizado o no cualquier intervención sobre la vía, se establecieron nuevos hitos con dedicatorias varias como por ejemplo con ocasión del viaje de un emperador a través 
de la provincia ${ }^{7}$, incluso en relación con sus celebraciones de cumpleaños o aniversarios, o simplemente de adhesión como demostración de lealtad (Cooley, 2012: 132) $)^{8}$. De esta forma, en numerosos casos los miliarios se convirtieron en verdaderos palimpsestos por acumulación de incripciones de los sucesivos emperadores (Kolb, 2015: 657-658).

Otra evidencia de cambio a partir de esta época es que se hace habitual el bilingüismo en sus textos ${ }^{9}$, o incluso la sustitución del griego por el latín (Van Tilburg, 2007: 20; Cooley, 2012: 167). Por ejemplo, en Judea durante el siglo II d. C. se redactaban sólo en latín, no obstante a partir de la dinastía severiana se añaden términos en griego a los ya preexistentes y después de Caracalla el latín desaparece de algunos de ellos (Isaac y Roll, 1982: 67-80, n. ${ }^{\text {os }} 3,5,8,11-12$, 16-19; Isaac, 1998: 63; Eck, 2009: 39). Era menos usual la traducción completa al griego de algunos de sus textos como, por ejemplo, nos ilustra uno de Septimio Severo en la primera milla desde Escitópolis en Judea; la decisión de traducir todos los títulos imperiales obedecería a la posición privilegiada que ocupaba, justo al salir de la ciudad (Isaac y Roll, 1982: $\left.73, \mathrm{n}^{\mathrm{o}} 10\right)$.

De igual manera, aunque hasta el reinado de Severo los elementos 'republicanos' en la titulatura imperial habían permanecido en los miliarios, sin embargo, en 198 d. C., por primera vez, la tribunicia potestas no es mencionada (Isaac, 1993: 308) ${ }^{10}$ y en adelante no se va a indicar de forma regular, mientras que, cada vez más, aparecen los cognomina de origen militar ${ }^{11}$. Esto ha llevado a B. H. Isaac (1993: 308) a plantear una participación activa de Septimio Severo en tales hechos ya que estas innovaciones no podrían haberse llevado a cabo sin el permiso del emperador, y lo pone en relación directa con las famosas palabras pronunciadas en su lecho de muerte para sus dos hijos, que Casio $(76,15,2)$ nos ha transmitido: «Permaneced unidos, enriqueced a los soldados y no os preocupéis de los demás». Aunque en un principio el ejército es el responsable de esta labor, lo que explicaría las características comunes en las diferentes provincias, en época de Septimio Severo también las propias ciudades van

7. Por ejemplo, algunos procedentes de Judea del año 129 y 198 coinciden con visitas imperiales a la región: Isaac y Roll (1982: 96); sobre otros de Septimio Severo relacionados con sus visitas a otras provincias: Isaac, (1998: 55).

8. Pekary (1968:, 16-22) plantea la hipótesis de que los textos que iban a ser inscritos eran comprobados por los gobernadores y enviados a la cancillería imperial para que fueran aceptados; incidiendo en lo mismo König (1973).

9. El bilingüismo en los miliarios es bastante habitual en el oriente griego, $c f$. Hirschfeld (1907) y Adams (2004: 209 y 223).

10. Una de las excepciones que confirman la regla en uno de Vespasiano: Isaac y Roll (1976).

11. Sobre los cognomina militares del emperador cf. Campbell (1984: 123-133). a encargarse de ello (Isaac, 1993: 308) ${ }^{12}$. La indicación de las distancias se hace tanto en latín como en griego, o solamente en griego, lo que demostraría que esta parte del texto fue elaborada por y para los habitantes de la zona (Isaac y Roll, 1982: 76), sin embargo, la titulatura imperial no se modifica, sigue apareciendo en latín como antes.

Así, en el siglo III d. C. la afirmación de la autoridad imperial se va a realizar de una forma casi colegiada, con la participación de todos los miembros de la domus divina. La asociación de los hijos al poder va a ser una garantía de continuidad dinástica, destinada a afirmar el poder del soberano y, con ello, la estabilidad del Estado (Christol, 1999: 344 y 350). De esta manera, formando parte de este esquema o programa imperial, una de las novedades más notorias durante este siglo va a ser la participación de las mujeres de la familia imperial en la difusión desplegada por el aparato palatino ${ }^{13}$. Una de las actuaciones fundamentales promovidas por la dinastía severiana fue una decidida utilización de la imagen de las mujeres de su familia, para difundir información, a partir de la cual los titulares del imperium se presentaban como la encarnación de la garantía de estabilidad, continuidad y seguridad de su gobierno, aunque este papel promocional no llegó a verse correspondido con el ejercicio de un poder real (Saavedra Guerrero, 2006: 719). En este sentido hay que destacar la actuación de Septimio Severo en relación a su 'autoadopción' en la dinastía Antonina, por la cual se convierte en «hijo de Marco Aurelio», y que implicó también a sus hijos, fundamentalmente al mayor, Caracalla, que pasó a llamarse a partir de 195, Marco Aurelio Antonino, pero también, en cierta manera, a su esposa Julia Domna, que al menos desde el 14 de abril de 195 (Kienast, 2004: 167) tenía el título de mater castrorum, el mismo que, veinte años antes, Faustina había recibido de su esposo Marco Aurelio (Birley, 2001: 178 y 254; Kuhoff, 1993). Esta iniciativa, entre otras, suponía claramente el inicio del establecimiento de un proceso dinástico, que fue publicitado a todos los habitantes del Imperio romano, a través de las monedas y de la epigrafía.

Así pues, la presencia de las emperatrices en los textos de los miliarios ha sido reconocida también como una novedad del período de los Severos (Christol, 1997: 134; 1999, 350). Sin embargo, esta constatación ya se hizo evidente a principios del siglo XX; Mary G. Willians hacía alusión a la aparición de Julia Domna en sus textos (Williams, 1902). Asimismo, en 1907 Otto Hirschfeld también resaltaba su presencia

12. Lo demuestra para Judea Levick (2000: 115).

13. Sobre la participación de la mujer en la propaganda imperial durante el siglo III, la mayor parte de los estudios se refieren a las mujeres de la dinastía severiana: Lusnia (1995); Baharal (1996); Kosmetatou (2002); Fernández Ardanaz y González Fernández (2006). 
argumentando que, con el emperador Septimio Severo, empiezan a aparecer las mujeres de la familia imperial, sobre todo en la parte oriental del Imperio (Hirschfeld, 1907: 179). Concretamente se refiere a Julia Domna y a Plautilla, aunque para esta Augusta mantiene sus dudas, ya que no lo afirma con rotundidad ${ }^{14}$ debido a su abolitio nominis. También cita a Otacilia Severa y a Herennia Etruscilla que aparecen en miliarios de Pannonia y África. En este sentido cabe destacar que en la historiografía son diversos los autores que han aludido al tema, normalmente sin profundizar y casi siempre refiriéndose a la «peculiaridad»y «rareza» de la aparición de mujeres de la familia imperial en este tipo de soporte ${ }^{15}$.

\section{LA PRESENCIA DE MUJERES EN LOS MILIA- RIOS DEL SIGLO III}

A finales del siglo II e inicios del III, con el acceso de Septimio al poder se va a originar un nuevo escenario en la sede imperial que permitirá que algunas emperatrices puedan tener una nueva forma de reconocimiento oficial. Hasta 235 d. C. las llamadas emperatrices sirias van a tener un indudable protagonismo en las labores de gobierno junto al emperador en calidad de esposa y madre, como fue el caso de Julia Domna; de madre como Julia Mamea con Severo Alejandro; o de abuela, Julia Mesa que lo fue de Heliogábalo y de Severo Alejandro. Otras, aunque jugaron un papel mucho menos determinante, también se vieron reflejadas en este nuevo escenario que se abría con el siglo; es el caso de Plautilla, esposa de Caracalla, que incluso fue nombrada Augusta a partir de los esponsales, antes de la celebración de su matrimonio, así se vio reflejada en los epígrafes (Christol y Th. Drew-Bear, 1995; Christol, 1997: 127-140; Christol, 2006: 29-30). Tras esta dinastía y a lo largo del siglo III d. C. otras mujeres de la casa imperial, siempre Augustae, aparecerán junto a sus maridos en estos monumentos que se habían convertido ya plenamente en elementos de autorrepresentación.

Aunque como estamos viendo el inicio de esta práctica comienza con los Severos, sin embargo, a partir de los datos que han llegado a nosotros, conocemos un precedente bastante peculiar en la dinastía de los Antoninos. Se trata de un documento ${ }^{16}$ en el que se conmemora la construcción de un tramo de calzada

14. Hirschfeld (1907: 179, nota 2): «vielleicht auch Fulvia Plautilla», refiriéndose a los n. ${ }^{\text {os }} 21$ y 22 del Anexo. Ver comentarios en Christol y Drew-Bear (1995: 76, nota 49). Se trata de CIL VIII 12271 y 12272.

15. Hay que dejar claro que cuando nos referimos a las mujeres de la familia imperial, aludimos a las que poseen el título de Augustas. La única excepción es Matidia, que además aparece sola en el epígrafe.

16. Anexo, nº 1. Cf. Pagano y Villucci (1991). en Suessa Aurunca ${ }^{17}$, concretamente siete millas, por parte de Mindia Matidia (Kienast, 2004: 127 y 130), conocida también como Matidia Minor (Bruun, 2010; Gualerzi, 2005; Cascella y Ruggi D’Aragona, 2012; Cascella, 2013; Cascella, 2014), hija de la Augusta Matidia, nieta de Marciana, que fue esposa de Trajano, hermana de la emperatriz Sabina, esposa de Adriano $\mathrm{y}$, por lo tanto, cuñada de Adriano y tía materna de Antonino Pío. Hay que destacar su absoluta singularidad $^{18}$ porque en él sólo viene reflejada Matidia. No aparece el emperador reinante, que en este caso era Antonino Pío, y además Matidia tampoco llegó a ser Augusta. $\mathrm{Su}$ excepcionalidad estaría justificada, en todo caso, por la importancia del personaje, que jugó un significativo papel y desarrolló una gran influencia en el entorno del trono imperial. Realmente se trata del testimonio de un acto evergético ${ }^{19}$, uno más, hacia la ciudad de Suessa por parte de Matidia la joven.

A las menciones de Williams y Hirschfeld, habría que añadir la de Erich Kettenhoffen quien, en 1979, recogía en su libro sobre las Augustas sirias una serie de miliarios en los que aparecían algunas mujeres de esta dinastía y además destacaba el hecho de que muchos de ellos eran bilingües (Kettenhoffen, 1979: 97). Concretamente de Julia Domna recogía 25 (Kettenhoffen, 1979: 97); en este trabajo presentamos un total de 39 y lo normal es que el número, a medida que surjan nuevos hallazgos, siga aumentando ${ }^{20}$. En ellos aparece junto a su marido y sus dos hijos, en unos pocos también Plautilla, y finalmente tras la muerte de Severo y Geta, junto a Caracalla ${ }^{21}$. En su caso, la mayoría proceden del ámbito balcánico-asiático, y además algunos de ellos también están redactados en griego. En la parte occidental del Imperio sólo se han encontrado en África ${ }^{22}$, concretamente en Numidia, Mauritania Caesariensis y Africa Proconsularis, que corresponden al gobierno de Caracalla como único

17. A éste se refieren Gordon et alii (1997: 213, nota 93) y además inciden en su excepcionalidad: «....and even more strikingly a unique example of a milestone bearing the name of a female member of the imperial family.»

18. Pagano y Villucci (1991: 288): «vero e propio unicum»; Cascella (2014: 77).

19. Sobre actos evergéticos de Matidia en Suessa $c f$. sobre todo Cascella (2014).

20. Además de los nuevos hallazgos, y aunque en nuestro trabajo hemos intentado realizar una recogida lo más exhaustiva posible, somos conscientes de que debido a la dispersión de las publicaciones habremos cometido omisiones. Sin embargo, los recogidos manifiestan una tendencia clara y creemos que son suficientes para demostrar nuestra hipótesis de trabajo.

21. Es con diferencia la emperatriz que más aparece. $C f$. Anexo, $\mathrm{n}^{\mathrm{o}} 2$ a 39, ambos incluidos.

22. Hirschfeld (1913: 729) y Murphy (1945: 91) hacían hincapié en el hecho de que, en África, del reinado de Septimio Severo sólo eran conocidos algunos procedentes de Mauretania Caesarensis. Actualmente sabemos que existen más, procedentes también de otras provincias africanas. 
emperador y en los que aparece la emperatriz junto a su hijo. Respecto al más antiguo por ahora ${ }^{23}$, se trata de uno, bilingüe, procedente de Lagina, en Caria ${ }^{24}$; sin embargo, la fecha que se planteaba, 196/197, correspondiente a la tribunicia potestas IIII de Severo no era correcta ${ }^{25}$. Posteriormente se corrigió la lectura, en realidad se trataba de la VIIII, y por lo tanto la fecha correspondería a 200/201. En cualquier caso, su presencia nos sirve para hacer evidentes las novedades que se van produciendo en la difusión de las ideas propagadas desde el centro del poder: Septimio Severo concibe el Imperio como su propiedad personal, y por lo tanto marca los monumentos públicos, incluidos, por supuesto, los miliarios que, a su vez, señalarían las calzadas, como posesiones privadas, y además admite a su esposa y a sus hijos también como copropietarios (Williams, 1902: 264). Esta nueva distinción se mantuvo más tarde con Julia Mamea, Mesa y otras Augustae, de tal forma que se las colocó en un lugar visible en una de las actividades centrales del Estado Romano. Y aunque esto no nos dice nada de su papel en la toma de decisiones, sí lo hace, sin embargo, al menos de la forma en la que ellas fueron percibidas ${ }^{26}$.

\section{RECAPITULACIÓN}

Como ya hemos podido precisar, obviando el caso de Matidia por las razones anteriormente aducidas, la presencia de mujeres de la familia imperial, más concretamente de las Augustae, en los miliarios coincidió prácticamente con el inicio del siglo III d. C. Por lo tanto, la emperatriz Julia Domna es la primera Augusta que inicia este proceso. Este título fue otorgado por primera vez por Augusto a su esposa Livia por vía testamentaria. Sus sucesores, en mayor o menor medida, lo concedieron a sus madres, esposas y hermanas. Algunas de ellas además desempeñaron un papel importante junto al emperador, sobre todo en el siglo III d. C., y su participación en el poder junto a éste, al menos desde el punto de vista del papel del fasto y la representación imperial, venía refrendada a través del título de Augusta en las

23. Hay que tener en cuenta que hablamos de aquellos que se pueden fechar por elementos internos.

24. Cf. Anexo, $\mathrm{n}^{\circ} 2$.

25. En la mayor parte de las publicaciones, incluso actuales, se mantiene la fecha correspondiente a 196/197: Williams (1902); Hirschfeld (1907: 719; Payson (1917: 16); Kettenhoffen (1979: 97, nota 172); Levick (2007: 68); Rowan (2013: 249, nota 25). Para la cronología correspondiente a la novena potestad tribunicia: Christol y Drew-Bear (1995: 79-81); Christol (1997: 135-136). En cualquier caso, sigue siendo el más antiguo.

26. A propósito de esto, $c f$. Murphy (1945: 80-100); así mismo, refiriéndose a Julia Domna (1945: 103-104) dice de ella que es la primera emperatriz que de forma oficial participa en la vida pública y además la primera que figura de forma destacada en los epígrafes, sin embargo, no comenta nada acerca de su inclusión en este tipo de obra pública. diversas inscripciones y monedas a lo largo del Imperio Romano. Esto tuvo su cénit en el siglo III d. C. en el que diversas mujeres alcanzaron cotas, no tanto de poder directo, como de influencia sobre el dinasta, como se puede ver claramente con las denominadas emperatrices severas, o incluso Zenobia de Palmira, aunque en este caso al margen de la legitimidad imperial. Muchas de estas mujeres pasaron a formar parte del paisaje epigráfico junto a sus esposos e hijos en los monumentos erigidos en el mundo romano, incluidos los que jalonaban los caminos. No obstante, la situación iba a cambiar en el siglo IV d. C., aunque la mujer no llegó a tener un papel tan significativo como el que se alcanzó en el siglo III d. C., hecho comprobado además en lo que se refiere a exposición pública en los diversos monumentos. Asímismo las concesiones del título de Augusta fueron cada vez más escasas, hasta tal punto que se puede decir que con la muerte de Constantino en el año 337 d. C. la práctica cayó en desuso (Holum, 1983: 31), aunque éste lo confirió en 324 d. C. a su madre, Helena, y a su mujer, Fausta, en un gesto de configuración dinástica, acompañado además de la promoción de su hijo Constancio II al rango de César. Sin embargo, sus hijos se quedaron sin herederos, al igual que su sobrino Juliano, y por lo tanto no tuvieron ocasión de emularlo. Pero éste no fue en el caso de Valentiniano I (muerto en $375 \mathrm{~d}$. C.), fundador de la dinastía siguiente, que no se lo otorgó a ninguna de sus dos mujeres (Marina y Justina), a pesar de que cada una le dio un hijo, Graciano y Valentiniano II; a éste último lo hizo Augusto en el curso de la vida de su madre. A finales del siglo IV d. C. precisamente, el nombramiento de Flaccilla, esposa de Teodosio, impresionó a sus contemporáneos como una innovación espectacular y como una vuelta a la práctica constantiniana de prestigio dinástico (Euseb. $V C$ 3, 47, 1). Sin embargo, esto no tuvo continuidad. Pero volviendo al tema que nos ocupa tras Julia Domna, que además de ser la iniciadora de esta costumbre fue la más representada en lo que se refiere a número de miliarios conservados, un total de 39 . De esta misma dinastía también ocuparon su espacio en estos monumentos Plautila, Julia Mesa, Julia Mamea y Salustia Orbiana, la única esposa de Alejandro Severo que tuvo el privilegio de aparecer en un miliario junto a la madre y la abuela de su marido, aunque por el momento sólo disponemos de un testimonio. A éstas siguieron Furia Sabinia Tranquilina, esposa de Gordiano III (238-244 d.C.); Otacilia Severa (26 miliarios, la segunda), esposa de Filipo el Árabe (244-249); Herennia Etruscila, esposa de Decio (249-251 d.C.); Cornelia Supera, esposa de Emiliano (jul./ag.-sept./oct. 253); Cornelia Salonina, esposa de Galieno (253-268 d.C.); Zenobia de Palmira, esposa de Septimio Odenato y madre de Vabalato; Ulpia Severina, esposa de Aureliano (sept. 270 - sept. 275) y finalmente Galeria Valeria, hija de Diocleciano y segunda esposa de Galerio, que es la única Augusta del siglo IV cuyo nombre aparece reflejado en algún miliario.

En definitiva, las mujeres de la familia imperial, generalmente esposas, tuvieron un protagonismo muy 
importante en la representación política durante el siglo III d. C., papel que se vio refrendado con su presencia, entre otros escenarios, en los miliarios. Como hemos podido documentar, una cantidad importante de mujeres, independientemente de su papel real en la corte, aparecieron reflejadas ante sus súbditos, en estos monumentos que jalonaban los caminos del Imperio. A partir del siglo IV d. C. la situación será otra; van a seguir existiendo, qué duda cabe, mujeres importantes, pero su papel va a ser muy distinto al desempeñado durante el siglo anterior y su número, en cualquier caso, va a ser mucho más escaso. De hecho, su ausencia en los miliarios no es más que un síntoma de la nueva situación que se va a producir a partir de la tetrarquía y sobre todo con la dinastía constantiniana.

\section{ANEXO. REPERTORIO DE MILIARIOS ${ }^{27}$}

\section{Mindia Matidia.}

\section{Latium et Campania}

1) Suessa Aurunca: MGR 1991, $287=$ AE 1991, 492. [Mati]dia Aug(ustae) | [fi]l(ia) | [divae S]abinae Aug(ustae) | [so]ror | [Imp(eratoris) Anto]nini Aug(usti) | [Pii p(atris) p(atriae) ma]tertera. (138-161).

2. Iula Domna (Kienast, 2004: 167-168), esposa del emperador Septimio Severo y madre de Caracalla y de Geta. A partir de junio de 193 Iulia Augusta.

\section{Iulia Domna, Septimius Severus, Caracalla y Geta.} Asia

2) Germencik: $A E$ 1896, $50=C I L$ III, $13689=S E G$ 45, 1594= RRMAM 3.5, 35 (pp. 81-82).

Iulia Domma mater castrorum.

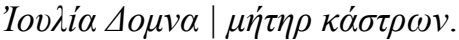

(200-201).

3) Doğruca: $A E$ 1888, $61=C I L$ III, 7179/80 $=A E$ $1995,1442=S E G 45,1669=$ RRMAM 3.5, 52(A) (pp. 105-106).

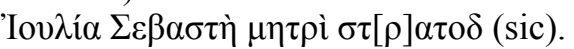

27. Para la confección de este anexo hemos utilizado las abreviaturas de corpora y los datos editados en el repertorio electrónico Clauss-Slaby por Manfred Clauss, cuya ingente y continuada labor en beneficio de la ciencia epigráfica hace mucho más fácil nuestro trabajo. Vaya desde aquí nuestro agradecimiento más sincero. En el apéndice, además, figuran dos numeraciones, la que se refiere a las emperatrices mencionadas (14 en total) y la que alude al número de cada uno de los miliarios (101 en total). En cada referencia hemos incluido el lugar del hallazgo (provincia romana y localidad actual), seguido de las ediciones; asimismo se incluye el nombre y títulos de cada emperatriz tal y como se reflejan en el texto original (latino y/o griego) y finalmente la cronología. También hacemos mención de los integrantes masculinos de la familia imperial que aparecen citados en cada epígrafe.
$(198-209)$.

4) Hacilar: $C I G$ 2, $3179=C I L$ III, 471-475 $=A E$ $1995,1466=S E G 45,1627=$ RRMAM 3.5, 57(A), (pp. 120-121),

Iulia [e Domnae Aug] | et matri [castrorum].

(201-202).

5) Akça: $A E$ 1976, 659 = SEG 26, $1365=$ RRMAM 3.5, 67 (B) (pp. 134-135).

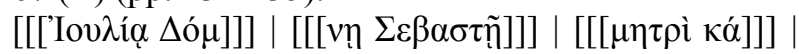
$[[[\sigma \tau \rho \omega v]]]$.

(198-209).

6) Gökçeler: $C I G 3,3857 d=I G R R P I V, 599=M A M A$ 10, $227=R R M A M 3.5,78$ (pp. 150-151).

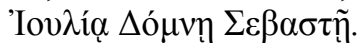

(193-209).

7) Gökçeler: $A E$ 1993, 1510 a-c $=$ RRMAM $3.578(\mathrm{C})$ (pp. 152-153).

'Iovגı́a | $\Delta$ ó $\mu v \eta\rceil$.

(198-209).

8) Alibey: $M A M A 10,93=A E$ 1993, $1509=R R M A M$ 3.5, 79 (pp. 153-154).

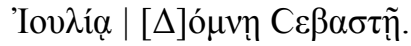

$(198-209)$

9) Bodrum, $S E G$ 16, $665=A E 1959,6=A E 1999$, $1593 \mathrm{a}=$ RRMAM 3.5, 114 (pp. 207-208).

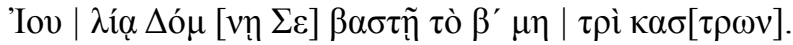
(200-201).

10) Çamköy: IGRRP IV, 925=IK 60, 14=RRMAM 3.5, 151 (pp. 274-275).

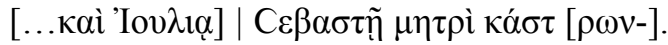
(198-209).

11) Gölhisar: $I K$ 60, $15=$ RRMAM 3.5, 153 (pp. 275-276).

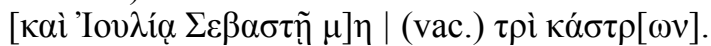
(198-209).

12) Gölhisar: IGRRP IV, $926=I K$ 60, $16=R R M A M$ 3.5, 154 (pp. 277-278).

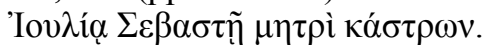

(198-209).

13) Büyük Alan: $I K$ 60, $17=R R M A M ~ 3.5,156$ (pp. 278-279)

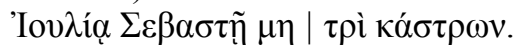

$(198-209)$.

14) Ece: IGRRP IV, $924=R R M A M ~ 3.5,157$ (pp. 279-280)

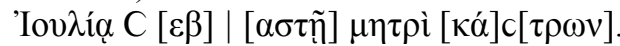
(198-209). 


\section{Lycia et Pamphylia}

15) Yar1: RRMAM. 3.6, 07(A) (pp. 33-34).

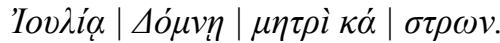

(198-209).

16) Deliktaș: RRMAM 3.6, 29(A) (pp. 65-66).

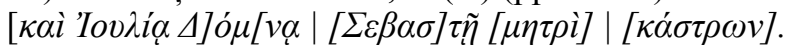
(198-209).

17) Deliktaș: RRMAM 3.6, 29(B) (pp. 65-66)

(¿Julia Domna?)

(198-209).

18) Çolaklı: $I K 44,176-177=A E$ 2001, $1922=R R$ MAM 3.6, 50(B), (pp. 98-99).

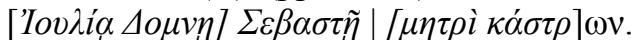

(198-209).

19) Alanya: RRMAM 3.6, 2(B) (pp. 101-102).

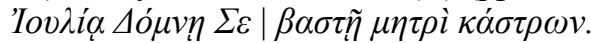

(198-209).

20) Demirtaș: IGRRP III, $826=A E$ 1965, $317=R R$ MAM 3.6, 53 (pp. 102-104).

'Iovגi $[\alpha \varsigma] \Delta o ́ \mu v \eta \varsigma|[\Sigma \varepsilon \beta \alpha \sigma \tau \tilde{\eta} \varsigma]|[\mu] \eta \tau \rho o ̀[\varsigma] \kappa \dot{\alpha}[\sigma]$ $\tau \rho \omega[v]$.

(198-209).

Iulia Domna y Fulvia Plautilla (Kienast, 2004: 165), esposa de Caracalla desde abril de 202 hasta enero de 205 (con Septimius Severus, Caracalla y Geta). Plautilla Augusta desde primavera-verano de $201^{28}$.

Asia

21) Bağc1lar: $C I L$ III, 482, 483 y add. (p. 982) $=A E$ $1995,1529=$ SEG 45, 1561= RRMAM 3.5, 112(A) (pp. 204-205).

Iulia Domna Aug mater castro / rum.

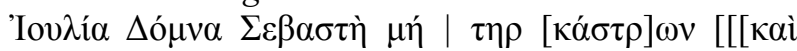

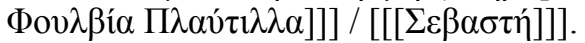

(200-201).

22) Bağc1lar: RRMAM 3.5, 112(B) (pp. 205-206).

Iul [D]o[mna Augusta] | [mater castr]orum.

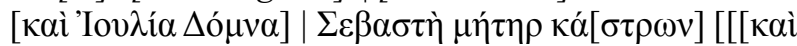

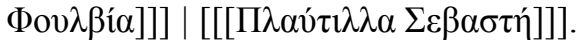

(200-201).

23) Sek: $A E$ 1991, 1511= $A E$ 1995, $1527=S E G 45$, $1526=$ RRMAM 3.5, 118(E) (pp. 218-220).

Iul(ia) Domna |A[ug mater cas] trorum [[[et Fulvia]]]

| [[[Plautilla Aug(usta)]]].

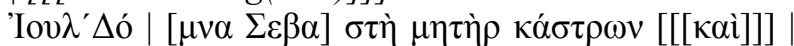

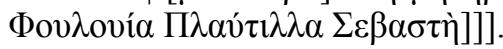

(200-201).
Septimius Severus y su familia (textos incompletos) ${ }^{29}$. Asia

24) Belevi: RRMAM 3.5, 7(E) (pp. 48-50).

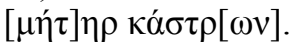

(200-201).

25) Pınarbaș1: $A E$ 1995, 1465 a-d $=S E G$ 45, $1627=$ RRMAM 3.5, 56(D) (pp. 118-119).

Texto griego.

(200-201).

26) Hac1lar: $A E 1995,1467=S E G 45,1627=R R M A M$ 3.5, 57(B) (p. 123).

Texto griego.

$(200 / 201)$

27) Kırkağaç: RRMAM 3.5, 98(A) (p. 181).

Texto griego y latino.

(198-209).

28) Kadıköy: $C I L$ III, $142014=S E G 45,1655=R R$ MAM 3.5, 145 (pp. 267-268).

Texto griego.

(198-209).

Iulia Domna y Caracalla.

Mauretania Caesariensis

29) Sitifis: $C I L$ VIII, $10340=$ CIL VIII, 22401.

Iuliae Dom(nae) Aug(ustae) mat | ris castror(um) et senat(us) et pa $\mid$ triae.

(214-215).

30) Sitifis: $C I L$ VIII, $10359=C I L$ VIII, $22403=A E$ 1937, 56

Iuliae | Domnae Aug(ustae) matr(i) castr(orum) et se | $n$ [at(us)] et patr(iae).

(214-215).

31) Thamallula: BCTH-1907-CXCI.

Iuli(a)e Dom $\{i\} n(a)$ e Aug(ustae) | [mat]ri castrorum et se | [nat]us et patri(a)e.

(214-215)

\section{Numidia}

32) Erkeb: $C I L$ VIII, $10197=C I L$ VIII, $10198=C I L$ VIII, $22326=A E$ 1946, 67.

[et Iuliae] | Aug(ustae) matr[i Aug(usti)] | et castro[rum] | et senatu[s et] | patriae.

(214-215).

33) Mascula: CIL VIII, 10202

[et Iul(iae) Aug(ustae)] ma[t(ri)] | [Aug(usti) et c] astro[r(um)] | [et sena]tus [ac] / [patriae].

(214-215). 
34) Lambaesis: CIL VIII, 10231

[Iuliae Aug(ustae)] | mat(ri) Aug(usti) et cas(trorum) et $s$ (enatus) | ac patriae.

(214-215).

35) Lambaesis: CIL VIII, 10253.

Iulia Augusta | matre Aug(usti) et | castror(um) et se|natus ac patriae.

(214-215).

36) Lambaesis: CIL VIII, 10260.

Iul(iae) Aug(ustae) mat[ri Aug(usti)] | et cas(trorum) et $\operatorname{se}[\mathrm{n}$ (atus) ac patri]|[ae.

(214-215).

37) Lambaesis: CIL VIII, 22358.

Iuliae Aug(ustae) mat(ri) | Aug(usti) et castr(orum) et | sen[at(us)] ac patriae.

(213-214).

38) Lambaesis: CIL VIII, 22359.

Iuliae Aug(ustae) matr(i) | Aug(usti) et castr(orum) et | sena(tus) ac patriae.

(214-215).

39) El Atech: CIL VIII, $10379=$ CIL VIII, 22413.

[et Iuliae] | Aug(ustae) m[at(re) Aug(usti) et] | [castr(orum) et sen(atus) ac pat(riae).

(215-216).

\section{Africa Proconsularis}

40) Thullium: CIL VIII, 22421.

Iuliae Au $[g($ ustae) $] \mid$ matri Aug(usti) et | castrorum | et senatus ac | patriae.

(213-216).

4 IULIA MAESA (Kienast, 2004: 181), hermana de Julia Domna, madre de Julia Soemias y de Julia Mamea, abuela de Heliogábalo y Severo Alejandro. Iulia Maesa Augusta, desde 30 de mayo de 218.

5 Iulia Avita Mamaea (Kienast, 2004: 180), madre de Severo Alejandro (222-235). Iulia Mamaea Augusta, poco después del 14 de marzo de 222.

6. GNaea Seit Herennia Sallustia Barbia OrbiaNA (Kienast, 2004: 179), esposa de Severo Alejandro. Sallustia Barbia Orbiana Augusta, después del 28 de agosto de 225 y antes del 30 de agosto de 227.

Iulia Maesa, Iulia Avita Mamaea, Sallustia Orbiana y Severus Alexander.

\section{Numidia}

41) Thamugadi: $A E$ 1981, 902.

Iulia Mamaea]]] | [[[Aug(usta) matre Aug(usti) n(ostri)]]] | et Iulia Maesa Aug(usta) matre castrorum et exer|citus [[[et Aug(ustae)]]] avia | [[[Aug(usti) n(ostri) et Cn(aea) Seia]]] Herennia?]]] Aug(usta) [[Se[veri Aug(usti) coniuge]]].
(225-227).

\section{Iulia Mamaea y Severus Alexander.}

Tracia

42) Hadrianopolis: IGBulg III, 2, $1827=I G R R P$ I $772=1892,11$.

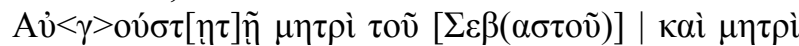

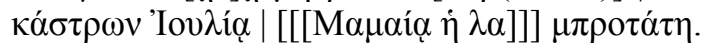

(222-231).

\section{Cilicia}

43) Yeşildam: $I K$ 56, 1, $14=$ RRMAM 3.7, 15(D) (pp. 32-33).

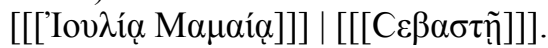

(222-223)

44) Tozlu: $S E G$ 12. $517=I K$ 56, 1, $13=R R M A M 3.7$, 16(A) (pp. 34-35).

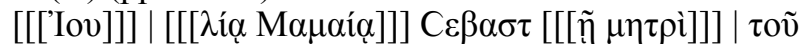

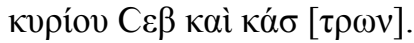

$(222-235)$.

45) Orta Tozlu: $I K$ 56, 1 = RRMAM 3.7, 17 (p. 37).

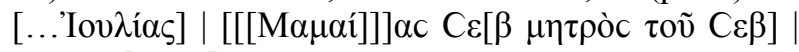

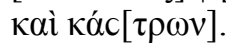

(222-235).

7) Furia Sabinia Tranguillina (Kienast, 2004: 197), esposa de M. Antonius Gordianus, Gordiano III (238-244 d.C.). Furia Sabinia Tranquillina Augusta, antes del 12 de mayo de 241 .

\section{Tranquillina y Gordianus.}

Moesia Superior

46) Ad Fines: $A E$ 1998, 1117.

Sabiniae | Tranquilli|[nae] Aug(ustae).

(241-242).

\section{Moesia Inferior}

47) Belimel: $C I L$ III, 14211,09 (p 2316,45) = ADBulgar $62=$ IDRE 2, 316=AE 1896, 116 .

Sabiniae Tranquil|linae sanctissima[e] | Aug(ustae) coniugi $\mid$ d(omini) n(ostri) | Gordiani.

(241-242).

\section{Cappadocia}

48) Sulusaray ${ }^{30}$ (Sebastopolis): CIL III, $6749=$ CIL III, $6894=$ CIL III, $14184=$ RRMAM 3.3, 61 (pp. 97-98). Sabi(nia) Tranqui | llina Aug(usta).

9 mayo (?) - 9 dic. 238.

8) Otacilia Severa (Kienast, 2004: 200-201), esposa de M. Iulius Philippus, Filipo el Árabe (244-249).

30. Cf. D. H. French (2012: 97-98). Erróneamente la base Clauss-Slaby la sitúa en Pontus et Bithynia: EDCSID:EDCS-28400806 
Marcia Otacilia Senera Augusta desde la primavera de 244.

\section{Otacilia y Philippus.}

Mauretania Caesariensis

49) Novi: CIL VIII, 10453 = CIL VIII, 22581.

Marciae Otaci | liae | Severae Aug(ustae) ma | tri castrorum.

(244-248).

\section{Pannonia Inferior}

50) Matrica, $A E$ 1969/70, 496.

Marc(ia) Otacilia | Severa sanctissima | Au(gusta) coniun [x] | Aug(usti) [n]ostri.

(244-248).

51) Intercisa, $A E$ 1969/70, 512.

[Marcia]e Otac[iliae S]ev[erae sanct(issimae)] ।

[Aug(ustae)] co[niugi Aug(usti) n(ostri).

(244-248).

52) Ulcisia: CIL III, 3641 = CIL III, 10654.

Marciae Otaci] | [liae Severae] sanc|tissima [e A] ug(ustae) co|ningi Aug[usti] | nostr [i.

(244-248).

53) Aquincum: CIL III, 3717 (p. 1717).

Marciae Otaciliae | Sever(a)e sanct[i]ssim(a)e | Aug(ustae) coniugi Aug(usti) n(ostri).

(244-248).

\section{Pannonia Superior}

54) Sutto: CIL III, 4627 = CIL III, 11334.

Marcia $\mid$ Otacilia Severa $\mid$ sanctissima Aug(usta) $\mid$ coniux Aug(usti) n(ostri).

(244-248).

55) Brigetio CIL III, 11326.

Marcia] | [Otacilia Se]vera | Sa[n]ctis[sima] Aug(usta) $\mid$ coniux Aug(usti) n(ostri).

(244-248).

56) Brigetio: CIL III, 11328.

[et Marc]ia Otacil[ia Severa.

(244-248).

\section{Moesia Superior}

57) Vrtiste: ILJug 2, 592 =IMS 4, $125=\mathrm{AE} 1980,792$.

Marc [iae Otaciliae] | Sever[ae sanctis]|[simae Aug(ustae) matri] | [castrorum.

(244-248).

Asia

58) Așağı Kırıklar: RRMAM 3.5, 21 (pp. 69-71).

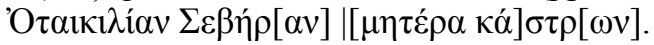

(244-248).

Otacilia, Philippus y Philippus (Iunior). Africa Proconsularis
59) Tunes: CIL VIII, 10049.

[et Marcia Otac]ilia [Seve]|[ra mater Caesaris et] | [castrorum et senatus] | [et patriae.

(244-248).

60) Musti: CIL VIII, $22089=$ ILTun 1732.

Marcia Otacilia | [Seve] ra Aug(usta) coniunx | Aug(usti) n(ostri) et mater Caes(aris) $\mid n$ (ostri) et castrorum et se|natus et patriae.

61) Lares: CIL VIII, $22127=$ ILTun 1732.

Marc[ia Ot]a|[cilia] S[evera] co | [niu]x [Aug(usti) n(ostri) mate]r se | na[tus et patriae et] | [castr]orum (244-248).

62) Thibursicum Bure: BCTH 1919, XXXI.

Ma |rcia Otacilia Se | vera Aug(usta) mater | Caesaris Aug(usti) et $\mid$ castrorum et $\mid$ senatus et patriae.

(Finales 246 - julio/agosto 247).

63) Jeddara: $A E$ 2012, 1897.

Ma|[rcia Otacilia Severa coniux Aug(usti).

(245).

\section{Aquitania}

64) Lugdunum Convenarum: CIL XVII, 2, $308=C I L$ XIII, $8889=C A G 31$, p 176 .

Ota $\mid$ ciliae Severae | Aug(ustae) ma(t)ri $\{u\}$ Aug(usti) | $n$ (ostri) et castror(um).

(244-248).

\section{Pannonia Inferior}

65) Aquincum: CIL III, 3718.

Marciae Otaciliae Severae | sanctissimae coniugi Aug(usti) n(ostri).

(244-248).

66) Aquincum: CIL III, 10627.

Marc[iae Otacili]ae Severae | sa[n]ct[issimae] Aug(ustae) | coniu[gi] Aug(usti) [n(ostri)].

(244-248).

67) Aquincum: CIL III, 14354,06.

Marciae Otaciliae | Severae sanctissimae |Aug(ustae) coniugi Aug(usti) n(ostri) | matri castrorum et | exercitu\{u\}s).

(244-248).

68) Puspokhalom: $A E$ 1973, 442.

Marcia Otacilia | Severa Aug(usta) coniu[x Aug(usti) n(ostri)].

(244-248).

69) Annamatia: CIL III, 10640

Marciae | Otacil[iae] Severae | sanctissimae Aug(ustae) n(ostrae) | coniugi Aug(usti) n(ostri) matri | castrorum exercitus.

(244-248). 
70) Matrica, AE 1969/70, 497.

Marcia | Otacilia Severa sanc | tissima Aug(usta) coniunx | Aug(usti) n(ostri).

(244-248).

\section{Pannonia Superior}

71) Csiv: CIL III, 04631.

Marcia Otacilia Se | vera Augusta [ma] | ter Aug(usti) n(ostri) et [cast] | rorum.

(244-248).

\section{Asia}

72) Tekin (Magnesia ad Maeandrum): CIL III, 12270

=IvMagnesia $257=I K$ 17, 1, $03164=$ RRMAM 3.5, 110 (p. 199).

Marciae | [S]everae Aug(ustae) matri castrorum.

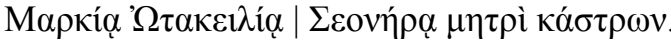

(244-248).

73) Hisar: RRMAM 3.5, 158 (p. 281)

Marciae O[taciliae Severae] | (vac) Aug [-]

(244-248).

\section{Cappadocia}

74) Çamsaray: $A E$ 1986, $654=$ RRMAM 3.3, 138 (pp. 239-240).

$M$ (sic) Otacil S[e] | verae Aug n et $m[a] \mid$ tri castrorum. (244-248).

9) Herennia Etruscilla (Kienast, 2004: 206), esposa de C. Messius Quintus Decius Valerinus (249-251 d.C.) Herennia (Cupressenia) Etruscilla Augusta desde otoño de 249

\section{Etruscilla y Decius. \\ Numidia}

75) Thamugadi: $A E$ 1981, 892.

Heren(n)iae | Etruscillae | con(iugi) Aug(usti) n(ostri). (250).

76) Thamugadi: BCTH 1951/52, 229.

Heren [ni]|ae Etruscil|lae coniu|gi Aug(usti) n(ostri). (249-251).

77) Lambafundi: BCTH-1951/52, 236.

Herenn[ia]e | Etruscillae | con(iugi) [Aug(usti) $n$ (ostri).

(250).

\section{Asia}

78) Aşağı Çă̆lan: $A E$ 1986, $00678=R R M A M 3.5$, 85(A) (pp. 160-161).

Erenniae | Etruscill|ae Aug(ustae).

(Sep./oct. 249 -250).

79) Belevi: $A E$ 1995, 1463= SEG 45, $1579=R R M A M$ 3.3, 7(D) (p. 47).

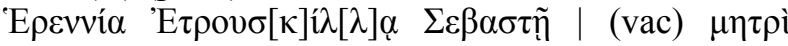

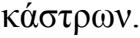

(¿Mitad de 250?).

80) Balçıkhisar: IGRRP IV. $771=$ RRMAM 3.3, 65 (pp. 130-131).

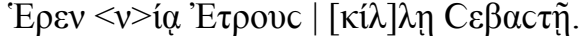

(Sept./oct. 249 - mayo/jun. 250).

81) Așağ1 Çağlan: $S E G$ 36. $1195=A E$ 1986. $678=$ RRMAM 3.3, 85(A), (pp. 160-161).

Erenniae | Etruscill |ae Aug.

(Sept./oct. 249 - 251).

82) Sek: $A E$ 1991, $1508=S E G$ 41, $939=R R M A M 3.3$, 118(A) (p. 214).

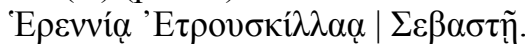

(Sept./oct. $249-251$ ).

83) Yakakayı: SEG 32. $1278=$ RRMAM 3.3, 1 (pp. 34-35).

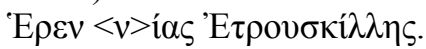

(Sep./oct. $249-$ sept. 251).

84) Balçıkhisar: IGRRP IV, $771=R R M A M ~ 3.3,65$ (pp. 130-131).

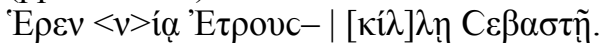

(Sep./oct. 249 - sept. 251).

\section{Pontus et Bithynia}

85) Çaycuma: RRMAM 3.4, 17(D) (pp. 53-54) [et H] erennia $<e>\mid$ [Etr] uscillae piae $\mid$ [feli]ci Augustae (?).

(¿249?).

Etruscilla, Decius, Herennius Decius y Hostilianus. Africa Proconsularis

86) Gamart: BCTH 1943/45, $104=A E$ 1942/43, 55.

Herennia Etruscil[la] | Aug(usta) coniunx Aug(usti) n(ostri).

(Mayo/jun. 250 - antes del 9 de junio de 251).

87) Gamart: $A E$ 1942/43, 55.

Herennia Etruscil[la] | Aug(usti) coniunx Aug(usti) n(ostri).

(Mayo/jun. 250 - antes del 9 de junio de 251).

Moesia Superior

88) Guberevac: IMS 1, $175=A E$ 1976, 605.

Heren[niae] | [Etru]scill(a)e.

(249-250).

89) Novi Bracin: IMS 4, 124

Here[nniae] | Etruscill(a)e [coniugi] | d(omini) n(ostri) Aug(usti) Imp[eratoris].

(249-250).

\section{Asia}

90) Serinhisar. RRMAM 3.5, 149(B) (pp. 270-272).

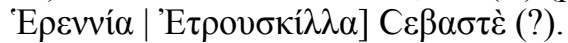

(249-251). 
10) Cornelia Supera (Kienast, 2004: 212-213), esposa de Aemilius Aemilianus (jul./ag. -sept./oct. 253). C. Cornelia Supera Augusta desde agosto de 253 (o algo más tarde).

\section{Cornelia Supera y Aemilianus.}

Numidia

91) Cuicul: $A E$ 1911, 104.

[[et C(aiae) Co[rne]liae [S]upe[r]ae Aug(ustae)]] | [[[con]i[u]gi]] eius.

(Ag./oct. 253).

11) Cornelia Salonina (Kienast, 2004: 222-223), esposa de P. Licinius Gallienus (253-268 d.C.). Cornelia Salonina Augusta desde 254 (¿?).

\section{Valerianus, Gallienus y Salonina.}

Asia

92) Așağı Kırıklar: RRMAM 3.5, 21 (2), (pp. 69-71)

[Corneliae Sal]oninae Aug(ustae).

(i254-jun.260?).

Gallienus y Salonina.

Africa Proconsularis

93) Qirrim, Wadi: IRT 927. (

[Saloni]n[ae] coniugis Gal(li)en $<i=O>$

(264).

Valerianus, Gallienus, Valerianus Iunior y Salonina. Numidia

94) Mascula: CIL VIII, $17680=C I L$ VIII, 22302.

Corneliae Salo | ninae Aug(ustae).

(257-258).

Valerianus, Gallienus, Valerianus Iunior, Saloninus y Salonina.

Galatia

95) Çağırkan: RRMAM 3.2, 69(B) (pp. 100-101)= AE 2012, 1563.

Saloninae matri | Augg(ustorum) et castror(um) | ux(ori) A(ugusti) p(iae) f(elicis) Aug(ustae).

(257-258).

12) Iulia Aurelia Zenobia (Kienast, 2004: 241242). Esposa de Septimius Odaenathus y madre de Vaballathus. (Septimia) (Septimia) Zenobia Augusta desde primavera de 272.

96) Palmyra: IGRPP III $1027=1065^{31}=$ OGIS $647=$ CIG 4503b.

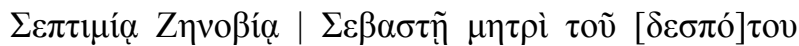

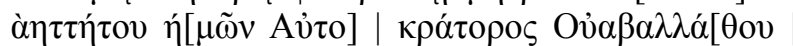

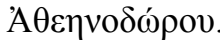

(272).

31. Entrada duplicada.
97) Vía Palmira-Emesa: IGRRP III, $1028=C I S$ II, $3971=$ OGIS 649

Bilingüe griego y palmireno.

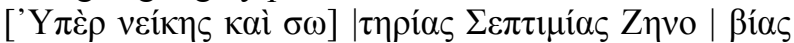

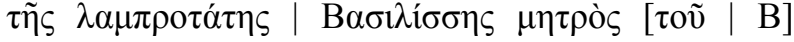

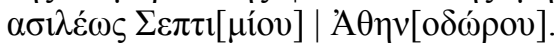

$(268-270)$.

98) Vía Palmira-Emesa: IGRRP III, $1029=$ OGIS 650

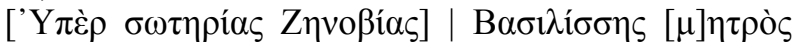

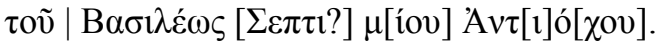

$(268-270)$

13) UlPia SeVErina (Kienast, 2004: 236-237), esposa de L. Domitius Aurelianus (sept. 270 - sept. 275). Ulpia (Severina) Augusta desde ca. 29 agosto de 274 (¿).

\section{Ulpia Severina y Aurelianus.}

Asia

99) Hac1lar: $C I L$ III, 471-475 IGR IV, $1482 \mathrm{c}=A E$ $1995,1466=S E G 45,1627=R R M A M 3.5,57(\mathrm{~A})(\mathrm{pp}$. 119-123).

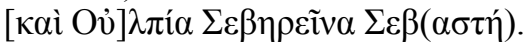

(29 ago. 274 - sept./oct. 275).

14) GaLeria VALeria (Kienast, 2004: 286-287), hija de C. Valerius Aurelius Diocletianus (284-305), segunda esposa de C. Galerius Valerius Maximianus (293-311). Galeria Valeria Augusta desde noviembre de 308 (i).

Diocletianus y Maximianus seniores Augusti; Galerius y Licinius I Augusti [;Galeria Augusta?] con Maximinus y Constantinus I filii Augustorum.

Asia

100) Yeniköy: IGRRP IV, $1364=S E G 54,1213=A E$ 2004, $1394=R R M A M 3.5,101(\mathrm{~A})$ (pp. 185-187).

Texto griego (¿Galleria Augusta?)

308-310 (?)

101) Büknüș: RRMAM 3.5, 131 (pp. 240-241),

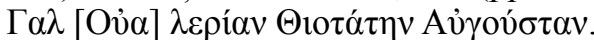

Nov. 308-310 (?).

\section{REFERENCIAS}

Adams, J. N. (2004). Bilingualism and the Latin Language. Cambridge.

Alföldy, G. (1991). Augustus und die Inschriften: Tradition und Innovation. Die Geburt der imperialen Epigraphik. Gymnasium, 98, 289-324.

Alves Dias, M. M. (2015). A consciência do valor propagandístico do texto epigráfico no Baixo Império. En Entre Roma e o Islão (pp. 93-102). Mértola: Campo Arqueológico de Mértola.

Ando, C. (2000). Imperial Ideology and Provincial Loyalty in the Roman Empire. Berkeley. 
Andreu Pintado, J. (2009). Tituli operum publicorum. En J. Andreu Pintado (Coord.). Fundamentos de Epigrafia Latina (pp. 397-463). Madrid.

Arce, J. (2007). Paul Veyne, L'Empire gréco-romain, Mélanges de la Casa de Velázquez, 37-1, 243-252. Recuperado de: http://mcv.revues.org/1845

Baharal, D. (1996). Victory of Propaganda. The Dynastic Aspect of the Imperial Propaganda of the Severi: The Literary and Archaeological evidence AD 193-235. Oxford.

Beltrán Lloris, F. (2015). Latin Epigraphy: The Main Types of Inscriptions. En Ch. Bruun y J. Edmondson (Eds.). The Oxford Handbook of Roman Epigraphy (pp. 89-110). Oxford.

Bender, H. (1989). Verkehrs- und Transportwesen in der römischen Kaiserzeit. En H. Jankuhn, W. Kimmig y E. Ebel (Eds.). Untersuchungen zu Handel und Verkehr der vorund frühgeschichtlichen Zeit in Mittel-und Nordeuropa: Bericht über die Kolloquien der Kommission für die Altertumskunde Mittel- und Nordeuropas in den Jahren 1980 bis 1983, V (pp. 108-154). Göttingen.

Birley, A. R. (2001). Marcus Aurelius. A biography. New York: Routledge.

Bruun, Ch. (2010). Matidia die Jüngere-Gesellschaftlicher Einfluss und dynastische Rolle. En A. Kolb (Ed.). Augustae. Machtbewusste Frauen am römischen Kaiserhof? Herrschaftsstrukturen und Herrschaftspraxis II. Akten der Tagung in Zürich 18-20.9.2008 (pp. 211-234). Berlin.

Cagnat, R. (1914). Cours d'Épigraphie Latine. Paris.

Calabi, I. (1968). Epigrafia Latina. Milano.

Campbell, J. B. (1984). The Emperor and the Roman Army. Oxford.

Cascella, S. (2013). Matidia Minore, la Bibliotheca Matidiana e il Foro di Suessa (Sessa Aurunca - Ce): considerazioni preliminari sullo scavo del cosiddetto Aerarium. Oebalus: studi sulla Campania nell'Antichità, 8, 147-217.

Cascella, S. (2014). Matidia Minor and Suessa Aurunca. En T. Opper (Ed.). Hadrian: Art, Politics and Economy (pp. 7388). London.

Cascella, S. y Ruggi D’Aragona, M. G. (2012). Memorie Suessane di Matidia. Suessa: Città e territorio dagli Aurunci all'età romana. Oxford.

Chevallier, R. (1999). Presentazione. En E. Banzi, I miliari come fonte topografica e storica. L'esempio della XI Regio (Transpadana) e delle Alpes Cottiae (pp. VII-VIII). Rome.

Christol, M. (1997). L'épigraphie de Thugga et la carrière de Plautien. En M. Khanoussi et L. Maurin (Eds.). Dougga (Thugga). Études épigraphiques (pp. 127-140). Paris.

Christol, M. (1999). L'épigraphie latine impériale des Sévères au début du IVe siècle ap. J.-C. En XI Congresso Internazionale di Epigrafia Greca e Latina, Roma, 18-24 settembre 1997, Atti (pp. 333-357). Rome.

Christol, M. (2006). L'Empire romain du III siècle: Histoire politique (de 192, mort de Commode, à 325, concile de Nicée). Paris.
Christol, M. y Drew-Bear, Th. (1995). Q. Aurelius Polus Terentianus et Q. Hedius Rufus Lollianus Gentianus, proconsuls d'Asie. Anatolia Antiqua, 3.1, 67-93.

Cooley, A. E. (Ed.) (2012). The Cambridge Manual of Latin Epigraphy, Cambridge: Cambridge University Press.

Donati, A. (2002). Epigrafia romana. La comunicazione nell "antichità. Bologna.

Eck, W. (2009). The presence, role and significance of Latin in the epigraphy and culture of the Roman Near East. En H. M. Cotton et alii (Eds.). From Hellenism to Islam: Cultural and Linguistic Change in the Roman Near East (pp. 15-42). Cambridge: Cambridge University Press.

Edcs: Clauss, M. (s, f). Epigraphische Datenbank Clauss Slaby. Recuperado de: http://www.manfredclauss.de

Encarnaçao, J. D’. (1995-1996). Miliários da Geira: informação e propaganda. Cadernos de Arqueologia, 12-13, $39-43$

Fernández Ardanaz, S. y González Fernández, R. (2006). El consensus y la auctoritas en el acceso al poder del emperador Septimio Severo. Antigüedad y Cristianismo, XXIII, 23-37.

Frei-Stolba, R. (1969). Inoffizielle Kaisertitulaturen im 1. und 2. Jahrhundert n.Chr. $M H, 26,18-39$.

French, D.H. (2012). Roman Roads and Milestones of Asia Minor. Vol. 3 Milestones. Fasc. 3.3 Cappadocia. British Institute at Ankara, Electronic Monograph 3. Recuperado de: http://biaa.ac.uk/publications/item/name/ electronic-monographs

Gordon, R., Reynolds, J., Beard, M. y Roueché, Ch. (1997). Roman Inscriptions 1991-95. Journal of Roman Studies, 87, 203-240.

Gualerzi, S. (2005). Una matrona sul confine. Matidia Maggiore. En L. Hernández Guerra (Coord.). La Hispania de los Antoninos (98-180): actas del II Congreso Internacional de Historia Antigua: Valladolid, 10, 11 y 12 de noviembre de 2004 (pp. 213-234). Valladolid.

Hirschfeld, O. (1907). Die römischen meilensteinen. Anhang: die gallischen Städtenamen auf den Meilensteinen. En Sitzungsberichte der Preußischen Akademie der Wissenschaften. Philosophisch-historische Klasse (pp. 165-201). Berlin: Verlag der Königlichen Akademie der Wissenschaften.

Hirschfeld, O. (1913). Kleine Schriften. Berlin.

Instinsky, H. U. (1938). Ein neuer Meilenstein von Pautalia und zwei Fragen zur Geschichte der Provincia Thracia. En Sitzungsberichte der Preußischen Akademie der Wissenschaften. Philosophisch-historische Klasse (pp. 1-10). Berlin: Verlag der Königlichen Akademie der Wissenschaften.

Isaac, B. H. y Roll, I. (1976). A Milestone of A.D. 69 from Judaea: The Elder Trajan and Vespasian. Journal of Roman Studies, 66, 15-19.

Isaac, B. H. y Roll, I. (1982). Roman Roads in Judaea, The Legio-Scythopolis Road, vol. I. Oxford.

Isaac, B. H. y Roll, I. (1996). Roman Roads in Judaea, The Jaffa-Jerusalem Roads, vol. II. Oxford. 
Isaac, B. H. (1993). The Limits of Empire. The Roman Army in the East (revised edition). Oxford.

Isaac, B. H. (1998). The Near East under Roman Rule: Selected Papers. Leiden.

Kenneth, G. H. (1983). Theodosian Empresses. Women and Imperial Dominion in Late Antiquity. Berkeley-Los Angeles-London: University of California Press.

Kettenhoffen, E. (1979). Die syrischen Augustae in der historischen Überlieferung: Ein Beitrag zum Problem der Orientalisierung. Antiquitas 3. Bonn: Abhandlungen zur.

Kienast, D. (2004). Römische Kaisertabelle. Grundzüge einer römischen Kaiserchronologie. Darmstadt.

Kolb, A. (2004). Römische Meilensteine: Stand der Forschung und Probleme. En R. Frei-Stolba (Ed.). Siedlung und Verkehr im Römischen Reich. Römerstrassen zwischen Herrschaftssicherung und Landschaftsprägung, Akten des Kolloquiums zu Ehren von Prof. H.E. Herzig vom 28. und 29. Juni 2001 in Bern (pp. 135-155). Bern.

Kolb, A. (2011): Miliaria: ricerca e metodi. L'identificazione delle pietre miliari. En F. Pavan (Ed.). I miliari lungo le strade dell'Impero. Aggiornamenti e nuove prospettive di ricercar (pp. 17-28). Verona.

Kolb, A. (2015). Communications and Mobility in the Roman Empire. En Ch. Bruun y J. Edmondson (Eds.). The Oxford Handbook of Roman Epigraphy (pp. 649-670). Oxford.

König, I. (1973). Zur Dedikation römischer Meilensteine. Chiron, 3, 419-427.

Kosmetatou, E. (2002). The Public Image of Julia Mamaea. An Epigraphic and Numismatic Inquiry. Latomus, 61(2), 398-414.

Kuhoff, W. (1990). Il riflesso dell'autorappresentazione degli imperatori romani nelle province dell'Africa (I-III sec. d.C.). L'Africa Romana, 7(2), 943-960.

Kuhoff, W. (1993a). Felicior Augusto Melior Traiano: Aspekte der Selbsdarstellung der römischen Kaiser während der Prinzipatszeit. Frankfurt.

Kuhoff, W. (1993b). Iulia Aug. mater Aug. n. et castrorum et senatus et patriate. ZPE, 97, 259-271.

Lassère, J.-M. (2005). Manuel d'épigraphie romaine, tomes 1 et 2. Paris.

Levick, B. (1982). Propaganda and the Imperial Coinage. Antichthon, 16, 104-116.

Levick, B. (2000). The Government of the Roman Empire. London and New York.

Levick, B. (2007). Julia Domna: Syrian Empress. London and New York.

Lusnia, S. S. (1995). Julia Domna's Coinage and Severan Dynastic Propaganda. Latomus, 54(1), 119-140.

Moreno, I. (2004). Vias romanas. Ingeniería y técnica constructive. Madrid.
Murphy, G. J. (1945). The Reign of the Emperor L. Septimius. Severus from the Evidence of the Inscriptions. New Jersey: St. Peters College Press.

Nicolet, C. (1988). L'Inventaire du monde. Paris.

Noreña, C. F. (2011). Imperial Ideals in the Roman West. Representation, Circulation, Power. Cambridge: Cambridge University Press.

Pagano, M. y Villucci, M. (1991). Un miliario di Matidia da Sessa Aurunca. MGR, XVI, 287-291.

Pekary, T. (1968). Untersuchungen zu den römischen Reichstrassen. Bonn.

Rowan, Cl. (2011). The Public Image of the Severan Women. Papers of the British School at Rome, 79, 241-273. DOI: https://doi.org/10.1017/S0068246211000031

Saavedra Guerrero, M. D. (2006). Augustae, uxores, mulieres et matres. Mujeres y ficción en la dinastía de los Severos. Mélanges de l'Ecole française de Rome. Antiquité, 118(2), 719-728. DOI: http://dx.medra.org/10.1400/78178

Salama, P. (1987). Bornes milliaires d'Afrique proconsulaire. Un panorama historique du Bas-Empire romain. Collection de l'École française de Rome 101. Rome: École française de Rome.

Salama, P. (1992). Anniversaires impériaux constantinoliciniens à Djemila. En Institutions, société et vie politique dans l'empire romain au IVe siècle après J.-C. Actes de la table ronde autour de l'œuvre d'André Chastagnol (Paris, 20-21 janvier 1989) (pp. 137-159). Collection de l'École Française de Rome 159. Rome: École française de Rome.

Santousso, A. (2001). Storming the Heavens: Soldiers, Emperors, and Civilians in the Roman Empire. Oxford.

Schneider, H.-C. (1982). Altstraßenforschung. Darmstadt.

Sillières, P. (1986). De la borne milliaire à la dédicace impériale. L'exemple de quelques inscriptions routières de l'Hispanie méridionale. REA, 88, 351-358.

Susini, G.C. (1982). Epigrafia Romana. Roma.

Van Tilburg, C. (2007). Traffic and Congestion in the Roman Routledge. London and New York.

Veyne, P. (2009). Objetivos del arte, propaganda y fasto monárquico. En P. Veyne. El Imperio Grecorromano (pp. 335-370). Madrid.

Wild Payson, S. (1917). Two Julias. The Classical Journal, 13(1), 14-24.

Williams, M.G. (1902). Studies in the Lives of Roman Empresses: I. Julia Domna. $A J A$, 6, 259-305.

Williams, M.G. (1904). Studies in the Lives of Roman Empresses: II. Julia Mamaea. En H.A. Sanders (Ed.). Roman Historical Studies and Institutions (pp. 67-100). New York.

Witschel, C. (2002). Meilensteine als historische Quelle? Das Beispiel Aquileia. Chiron, 32, 325-93. 